\title{
I MANOSCRITTI ARABI, PERSIANI E TURCHI DELLA BIBLIOTECA BRAIDENSE
}

\author{
AGOSTINO SOLDATI (*) \\ Nota presentata dal s.c. Claudio Gallazzi
}

(Adunanza del 9 giugno 2016)

SUNTO. - La nota si incentra sui manoscritti in carattere perso-arabico conservati presso la Biblioteca Nazionale Braidense, con breve descrizione degli esemplari di maggiore interesse e notizie sulla loro disparata provenienza. Del poco noto fondo l'autore attende alla redazione di un catalogo completo.

$$
* * *
$$

ABSTRACT. - The paper offers a survey of the manuscripts in Perso-Arabic characters kept in the Biblioteca Nazionale Braidense, with a brief illustration of the most substantial items and some information on their diverse provenance. The author is preparing an exhaustive catalogue of this little-known fund.

Secondo il normativo World Survey of Islamic Manuscripts di Geoffrey Roper, del 1993, la consistenza del fondo di manoscritti islamici conservato presso la Biblioteca Nazionale Braidense ammonterebbe a 23 esemplari. Tale computo riprende, evidentemente senza alcuna verifica, il risultato del censimento effettuato da Giuseppe Gabrieli nell'ancora prezioso repertorio Manoscritti e carte orientali nelle biblio-

(*) Sapienza Università di Roma, Dipartimento di Storia Antropologia Religioni Arte Spettacolo, Roma, Italia. E-mail: agostino.soldati@uniroma1.it 
teche e negli archivi d'Italia, dato alle stampe nel 1930. ${ }^{1}$ Già nel 1993, infatti, la Biblioteca di Brera possedeva gli attuali 27 manoscritti in caratteri perso-arabi accanto a un'esigua ma interessante raccolta di documenti in arabo e in turco ottomano. Non risulta legittimo definirli nel loro complesso 'manoscritti islamici' annoverandosi tra essi ben 11 manoscritti arabi cristiani.

Questo compatto gruppo costituisce forse il nucleo più antico del fondo orientale braidense, risalente con buona probabilità alla preesistente raccolta libraria gesuitica, o, al più tardi, frutto della soppressione napoleonica delle congregazioni religiose. A questo primo mannello si aggiunsero, nei decennî seguenti, 7 manoscritti arabi islamici, 2 manoscritti persiani, 1 manoscritto arabo-turco, 1 manoscritto persoturco, e 5 manoscritti turchi. La provenienza di questi 16 esemplari è assai composita: per tacere di isolate donazioni di privati o di altre istituzioni, alcuni tra i pezzi di maggiore interesse appartengono al lascito di un orientalista di notevole statura, ancorché oggi pressoché del tutto dimenticato, non fosse stato lontano parente di Carlo Cattaneo: alludo ad Antonio Madini, nobiluomo trentino vissuto tra il 1800 circa e il 1859 , l'ammirazione per il quale ancora era ben viva in Graziadio Isaia Ascoli e in Michele Amari. Di Madini la Braidense conserva anche non pochi stampati di argomento orientalistico, tra i quali rare copie delle sue stesse opere, come la traduzione commentata del capitolo dedicato al Siğistān del Kitāb al-masālik wa-al-mamālik del geografo arabo di sec. X Abū Isḥāq al-Ișțahrīī edita a Milano nel 1842. ${ }^{2}$

1 G. Roper, World Survey of Islamic Manuscript, II, London, Al-Furqān Islamic Heritage Foundation, 1993, 88; G. Gabrieli, Manoscritti e carte orientali nelle biblioteche e negli archivi d'Italia. Dati statistici e bibliografici delle collezioni, loro storia e catalogazione, Firenze, Leo S. Olschki, 1930 (Biblioteca di bibliografia italiana, X), 26.

2 Per la parentela con Carlo Cattaneo, cf. Carteggi di Carlo Cattaneo, Serie I. Lettere di Cattaneo. 1820 - 15 marzo 1848, a c. di M. Cancarini Petroboni \& M. Fugazza, Firenze \& Bellinzona, Le Monnier \& Casagrande, [2001], 524; godette di larga stima presso i contemporanei, cf. almeno G.I Ascoli, Note letterario-artistiche minori durante il viaggio nelle Venezie, nella Lombardia, nel Piemonte, nella Liguria, nel Parmigiano, Modenese e Pontificio. Maggio-Giugno 1852, a c. di S. Timpanaro, Annali della Scuola Normale Superiore di Pisa, II ${ }^{a}$ s., XXVIII, (1959), 151-191: 164, con n. 2. $\mathrm{Fu}$ autore per lo più di pregevoli traduzioni: Il Segistan, ovvero Il corso del fiume Hindmend secondo Abu Ishak-el-Farssi-el-Isstachri geografo arabo, Milano, Bernardoni, 1842, e Le rarità dell'istoria. Estratto di Maometto Anbaritano scrittore arabo recato dall'originale in italiano, Milano, Bernardoni, 1844. Così del Madini scriveva un 
Al 1892 risale, invece, l'oculato acquisto dei due manoscritti arabi di maggior pregio dell'intera collezione, compiuto grazie ai buoni uffici dell'allora prefetto della Biblioteca Ambrosiana, Antonio Maria Ceriani, mentre solo nel 1944 la Braidense poté assicurarsi i due sontuosi manoscritti persiani che oggi vanta, grazie alla munificenza del conte Daniele Castiglioni di Caronno, che adornò, come è ben noto, la biblioteca della cospicua raccolta di codici miniati e incunaboli riunita nel fondo a lui intitolato. ${ }^{3}$ La collezione di manoscritti in carattere perso-arabo ora sommariamente descritta è a tutt'oggi inedita, se si eccettuano le descrizioni dei due manoscritti persiani del Fondo Castiglioni e del perso-turco doverosamente accluse da Angelo Michele Piemontese nel Catalogo dei manoscritti persiani conservati nelle biblioteche d'Italia, dato alle stampe nel $1989 .{ }^{4}$

I due poziori manoscritti arabi cui si accennava (AG. XIV. 47 e AG. XIV.48) sono accompagnati da una scheda autografa di Ceriani, che con la consueta dottrina ve ne illustra contenuto e presumibile datazione:

Il Ms. più piccolo contiene il Divano di Baba al Din Abu' $l \mid$ Fadhal Ibn Zubair Ibn Muhammad Ibn Ali al I Muballabi.| Il titolo però scritto nel primo foglio è di mano e in carta | più recente del Ms. I Le poesie sono disposte in ordine alfabetico: presa per norlma alfabetica la rima finale dei versi. In fine, se manca l qualche foglio, deve essere poco quel che manca, la finale dei I versi essendo l'ultima lettera dell'alfabeto, sotto la quale $\mid c^{\prime} e$ e $\left[v^{\prime}\right.$ è $]$ già un discreto numero di carmi. | Il titolo del Divano dato dal codice del Sig. Caprotti [[Casati]] è più I completo che quello dato da altri Mss. da Casiri, Bibl. I Ar. Hisp. $n^{i}$ 443, e 475, da Dozy Cat. Codd. Mss. Arabb. Bibl. Lugduno Batavs $n^{\circ}$ DXXIX, e del

contemporaneo, G. Gherardini, Lessigrafia italiana proposta da G.G., Milano, co' tipi di Luigi di Giacomo Pirola, 1849, 92: “(...) Antonio Madini, conoscitore il più profondo (siccome e' fu già da altri qualificato) che a' dì nostri per avventura possegga l'Italia, delle lingue semitiche e soprattutto dell'araba, mi accerta che bizeffe è realmente voce arabesca (...)".

3 Notizie sulla donazione della collezione, acquisita presso la libreria antiquaria Hoepli, in A. Cutolo, I romanzi cavallereschi in prosa e in rima del fondo Castiglioni presso la Biblioteca Braidense di Milano, Milano, Istituto di biblioteconomia e bibliografia "Ulrico Hoepli" - Università di Milano, 1944, V-VII.

4 A.M. Piemontese, Catalogo dei manoscritti persiani conservati nelle biblioteche d'Italia, Roma, Istituto poligrafico e Zecca dello Stato-Libreria dello Stato, 1989 (Indici e cataloghi. Ministero dei beni e delle attività culturali e del turismo. Nuova serie, 5), 176-177. 
Catal. Codd. Mss. Arr. qui I in Museo Britannico asservantur Pars II Codd. Arabb. No MDCLXIX. I Dalla descrizione che hanno questi cataloghi dell'opera e dei 1 titoli, che variano in casi accidentali, ma si accordano nella | sostanza l'opera è la stessa in questi mss. e nel ms. Caprotti [[Casati]] I nonostante le varianti indicate nel principio della Prefazione nel Ms. di Londra, e nei due del Casiri, e nel [[?]] ms I Caprotti, perché quel di Londra varia dagli altri due, e tutti $\mid$ e tre dal nostro. I Il poeta mori nell'anno 656 dell'Egira, 1258 di Cristo, era d'Elgitto, e scrive in questo divano, come nota anche Dozy poesie I in lode di Saladino e di altri principi. La data apposta l a varii carmi, varia, ma coincide colla età del poeta. I Per determinare però più giustamente l'identità dei varii I Mss bisognerebbe confrontarli, o almeno avere estratti, che I non danno $i$ varii cataloghi. I Di quest'opera oltre gli indicati ci sono alcuni altri Mss. II legatura moderna. Il secondo Ms. mutilo nel principio e alla fine è egualmente | una raccolta di poesie disposte alfabeticamente come nel I primo. Da questa disposizione si vede che manca della I prima lettera, di parte della seconda e di parte dell'ultilma II Il titolo che manca in principio del Ms. mutilo è scritto I sul dorso superiore semplicemente ديوان ابن المقرب Divano di Ibn al Mukarrab. Nel Catalogo del | Mus. Britannico sopra citato $n^{\circ}$ DCVIII è descritto un Ms. I miscellaneo che comincia con questo Divano col nome I più pieno dell'autore Ali Ibn Mukarrab al Ujuni. I In questo Ms. però le poesie non sono disposte con ordine I alfabetico; esso è recente essendo del sec. XVII circa, mentre I quello Caprotti pare molto più antico. I L'autore scrive tra il 599 e il 618 dell'Egira, | 1201-1221 di Cristo, secondo la nota del Catalogo del Museo I Britannico, e l'uno o l'altro degli anni di questo periodo I sono notati talora anche nel Ms. Caprotti. I carmi | sono specialmente in lode di emiri Ujunidi, ma ce I $n ' \grave{e}$ anche pel Califfo di Bagdad, ed altri personaggi. I E da notare che secondo questo $\mathrm{Ms}$. al $\mathrm{fgl}$. o0 (55v) parrebbe I che l'autore scrivesse anche nell'anno 627 dell'Egira, e in I altri due luoghi è dato l'anno dell'Egira 626; se è esatta la I scrittura parrebbe doversi correggere l'anno dato sopra 618. I Questo poeta fu molto stimato da' suoi, e dai Wababiti | Nel codice Caprotti non ho trovato l'unica poesia, di cui si | cita il principio nel Catalogo del Museo Britannico, o perché I omessa, o perché poteva essere in fine del codice. Ma il soggetto I dei carmi nei due Mss. si accorda molto bene per quanto I si può desumere dalla breve descrizione. I I codici di questo poeta paiono vari. I I due Mss. sono in legatura orientale.

Dalle competenti note stilate dal prefetto dell'Ambrosiana si apprende come almeno il più antico dei due manoscritti, AG. XIV.48, appartenne al ricchissimo lotto di codici che il mercante lombardo 
Giuseppe Caprotti (1862-1919) - attivo per molti anni a Șan'ā', ai tempi suddita dell'impero ottomano - portò con sé a Milano; la maggior parte di essi poté assicurarsi, come è noto, la Biblioteca Ambrosiana grazie alla munificenza di banche e notabili milanesi. Si tratta di un ms. cartaceo del sec. XV contente, come informa il seriore titolo (f. 1r), il canzoniere di Bahā' ad-dīn Zuhayr. Di famiglia egiziana, quello che è considerato il massimo poeta di età ayyūbide nacque alla Mecca nel 581è./1187 e trascorse gli anni della formazione nell'alto Egitto, a Qūš. Trasferitosi nel 625è./1227 al Cairo entrò al servizio di aṣ-Șālih Ayyūb, figlio del sultano al-Kāmil, assurgendo ben presto alla carica di șạhị dīwān al-inšă', e, una volta che aṣ-Șālih Ayyūb ascese al sultanato, a quella di wazīr. Nel tempo in cui San Luigi guidava i Crociati all'invasione del Delta (646 è./1248), per un malinteso con il suo protettore, Bahā' ad-Dīn Zuhayr cadde in disgrazia; trascorse l'ultimo decennio della vita in solitudine e miseria. Morì nel 656 è. / 1258 al Cairo, oscura vittima della peste..$^{5}$ Il manoscritto, tra i testimoni più antichi che se ne conservino, reca il dīwān nella sua interezza in una copia di ragguardevole bontà, come appare dalla collazione col testo stabilito da Edward Henry Palmer, integrato dalla preziosa silloge critica di Stanislas Guyard e dalle moderne vulgate mediorientali.

Il secondo manoscritto acquistato grazie all'interessamento di Ceriani è AG.XIV.47, cartaceo, in scrittura nash̆ $\mathrm{i}$ ad inchiostro nero, è

5 The Poetical Works of Beha-ed-din Zoheir, of Egypt, with metrical English translation, notes, and introduction, by E. H. Palmer, 2 voll., Cambridge, at the University Press, 1876; S. Guyard, Le Divan de Beha ed-Din Zoheir. Variantes au texte arabe, Paris, Maisonneuve \& C $\mathrm{C}^{\mathrm{ie}}$ Libraires éditeurs, 1883; Ibn Khallikan's Biographical Dictionary, transl. from the Arabic by $\mathrm{B}^{\mathrm{n}}$ Mac Guckin De Slane, vol. I, Paris, Orient. Transl. Fund of G.B. and Irl., 1893, 542-545; C. Brockelmann, Geschichte der arabischen Litteratur, I, Leiden, E.J. Brill, 19432, 307 s.; Id., Geschichte der arabischen Litteratur, Erster Supplementband, Leiden, E.J. Brill, 1937, 465 s.; J. Rikabi, La poésie profane sous les Ayyûbides et ses principaux représentants, Paris, Librairie Orientale et Américaine G.-P- Maisonneuve \& C ${ }^{\circ}, 1949$, 121-164; Dīwān al-Bahä’ Zuhayr, šarḥ wataḥqīq Muḥammad Ṭāhir al-Ğabalāwī \& Muḥammad Abū al-Faụl Ibrāhīm, al-Qāhira, Dār al-Ma'ārif, [1977]²; J. RIKABI, Bahā' al-Dīn Zubayr, EI² I, 940a-b; P. Smoor, Bahā' al-Din Zuhayr, in Encyclopedia of Arabic Literature, ed. by J. S. Meisami \& P. Starkey, London \& New York, Routledge, [1998], 127a-b; Salma Khadra Jayyusi, Arabic poetry in the post-classical age, in Arabic literature in the post-classical period, ed. by R. Allen \& D.S. Richards, Cambridge, University Press, [2006], 25-59: 47-50. 
databile al sec. XVII (un sicuro terminus ante quem offre una data del 1107è./1695 che si legge in una delle note che affollano il f. 17r, risparmiato bianco dal copista). Mutilo del principio e della fine, conserva il canzoniere del poeta Ahmad bin 'Alī bin al-Muqarrab, della prosapia degli 'Uyūnidi del Bahrayn: qui tra il 1077 e il 1078 il capostipite della dinastia, 'Abdullah bin 'Alī al-'Uyūn̄̄, cacciatine i Qarmați, si era insediato quale vassallo degli 'Abbāsidi. Nato nel 572è./1178 ibn Muqarrab fu poeta di corte al tempo di Muḥammad bin Aḥmad bin Muhammad bin Faḍl e del figlio Mas'ūd. In séguito a dissapori con quest'ultimo lasciò la madrepatria: Yāqūt racconta di averlo incontrato a Mōṣul nel 617è./1220. Morì a Bag̉dād nel 629è./1232. ${ }^{6}$

Il gruppo di manoscritti arabi di contenuto cristiano prima ricordati si presenta abbastanza omogeneo: vi si annoverano evangeliarî, eucologi e florilegi di omelie; tutti i manoscritti, in sciolta scrittura nash $\bar{\imath}$ ad inchiostro nero e rubriche rosse, presentano caratteristiche bibliologiche affini. Pare verisimile siano stati esemplati in una medesima missione cattolica in Medio Oriente. Un indizio potrebbe fornire AG.XI.16, cartaceo, contenente la riduzione araba del catechismo di Bellarmino. Nella nota iniziale (f. 2v Fig. 1) si legge:

bi-'sm al-äb wa-'l-ibn wa-'r-rūh al quds al-ilah al-wāhid amīn | naktub kitāb ta' 'lìm al-masīḥi al-mu'allaf li-ağl I manfa'at awlād al-masīhiyyīn wa-ta'tìmibim haqā'iq I al-ìmān wa-buwa muqsam ilà arba'at aqsām ayyi fìmā yağib 'alà | al-masīhñ an yūfura bi-hi wa-ya lamahu wayatanāwalabu wa-yatlubahu lwa-qad tutarğam min al-lugiat al-fasransāwiyyah (1. faransāwiyyah) ilà al-lugiat al-'arabiyyah | bi-sa'ī wa-ta'ab liahad ar-rabbān al-karmalitāniyyīn al-hāniyyīn | al-mursalīn al-qätiniñn fì madīnat halab ǎs-šabbāa(') | fì sanat tamāniyat (1. tam-) | 'ašara wa-sab'umaiyah $\mid$ ba'd al-alf $\mid$ li-sayyidinā $\mid$ al-masīh.

Nel nome del Padre, del Figlio e dello Spirito Santo, il Dio unico, amen. Scrivo un libro di dottrina cristiana compilato per l'edificazione dei

6 Brockelmann, Geschichte der arabischen Litteratur, I, 302; Id., Geschichte der arabischen Litteratur, Erster Supplementband, p. 460; Dīwān Ibn al-Muqarrab, tahqīq wa-šarḥ 'Abd al-Fattāḥ Muḥmmad al-Ḥulw, [al-Qāhira], Maktabat at-Ta'āwun atTaqāīi, 1383 èg./1963 ; Salah Niazi, An Edition of the Dīwān of 'Alī Ibn Muqarrab accompanied by a Critical Study, 2 voll., diss. London, 1975. Sulla dinastia 'uyūnide, G.R. Smith, 'Uyünids, EI X, 960a; G. Rentz \& W.E. Mulligan, al-Bahrayn, EI I, 941a944b: $942 a-b$. 
figli dei Cristiani e l'istruzione loro ai dogmi della fede; esso è diviso in quattro parti che (vertono) sui doveri del Cristiano di crescere in essi, di conoscerli, di comprenderli e di indagarli. (Il libro) è stato tradotto dalla lingua francese in lingua araba grazie all'interesse e alla fatica di uno dei frati Carmelitani compassionevoli missionarî residenti nella città di Aleppo la grigia nell'anno settecento diciotto dopo il mille di Nostro Signore il Messia.

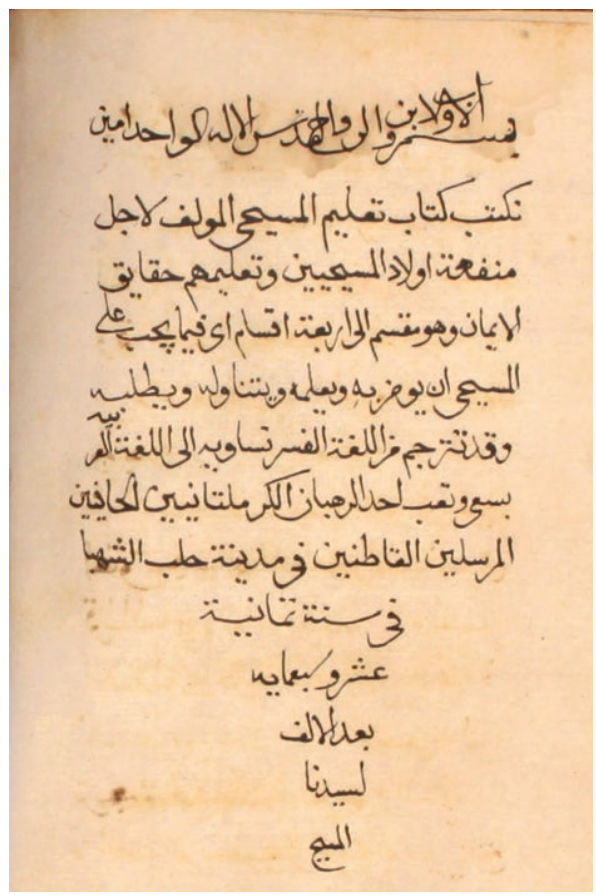

Fig. 1 - AG.XI.16, f. 2v.

Ceduto alla Braidense dalla Soprintendenza alle Belle Arti di Milano nel 1930, il raffinato AC.VIII.12, è il più sontuoso fra i tre Corani posseduti dalla biblioteca. Cartaceo, tipico prodotto di un atelier ottomano di tardo Settecento, di particolare nitore è la decorazione dei ff. $1 \mathrm{v}-2 \mathrm{r}$, ove le prime due sure sono ospitate in due medaglioni incastonati entro una doppia composizione simmetrica, coronati e seguiti da pannelli a decorazione geometrica con cartiglio centrale dalla scritta in bianco. Il tutto è delimitato da un festone fiorito a dominante blu. 
Generoso l'impiego di oro. Un identico medaglione ospita nell'ultimo foglio (f. 152v Fig. 2) un verboso colofone, che informa dell'identità del copista e della data di confezione del volume:

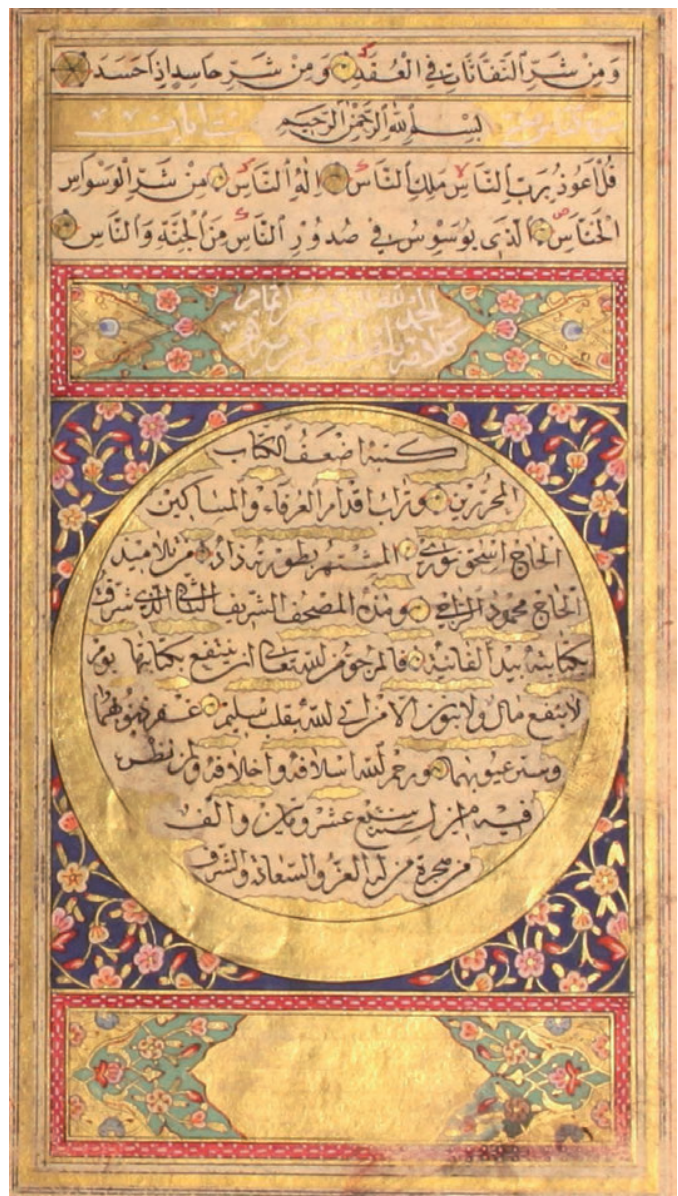

Fig. 2 - AC.VIII.12, f. 152v.

katababu ad'afu 'l-kuttāb | al-muharrarin (vel muharrirīn) • wa-tarābu

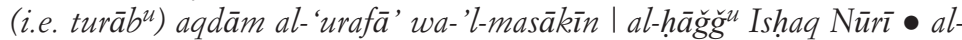

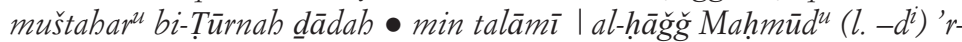

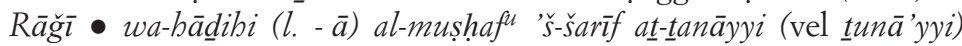
allad̄i šurriftu | bi-kitābatibi bi-yad al-fāniyyah $\bullet$ fa-'l-marğuww min Allāh ta' 'àlà an yantafi' bi-kitābatib̄ā yawm I lā yanfa' mālun wa-lā 
banūn illā man' 'atà 'llāb bi-qalb sulìm (salīm) • gafara unūbibumā | wa-satara 'uyūbibumā • wa-rahima Allāh aslāfahu wa-ahlāfahu wa-liman nazrabu | fïbi, amīn. li-sanat sab' 'ašr wa-mi'atayn wa-alfun| min hiğrab man lahu al-'izz" wa-'s-sa' 'ädab wa-'š-šaraf.

L'ha scritto il più umile degli scrivani I consacrati a Dio, polvere dei passi dei maestri e dei mendicanti, I il hạğğ Ishaq Nūrī, conosciuto come Torneh-zadeh, dei discepoli del hăğğğ Mahmūd ar-Rāğì. Questo è il secondo Corano (lett. 'nobile codice') della cui copiatura con mano caduca sono stato onorato. Ed è mia speranza in Dio l'Altissimo che dall'averlo copiato io tragga vantaggio "nel giorno in cui a nulla valgono le ricchezze e la prole se non per colui che giunge a Dio con cuore sincero (Cor. XXVI 88s.)". Rimetta i peccati del maestro e dell'allievo, faccia velo alle mancanze di entrambi, sia benigno Iddio verso gli avi e $i$ discendenti e chi avrà riguardo di questo volume, amen. Nell'anno mille duecento diciassette dell'ègira (1802) di Colui che detiene l'onore, la felicità e la gloria.

La sezione persiana del fondo, come si è detto, è limitata a due manoscritti pur di non comune pregio che la Braidense deve al Castiglioni, prospero costruttore lombardo. Dei due è doveroso soffermarsi almeno sul ms. FONDO CASTIGLIONI 22: di fattura iraniana, risale ai decennî centrali del sec. XVI; si tratta di un lussuoso esemplare della pentalogia poetica (Hamsah) di Nizāāmī. Particolarmente i bifogli iniziali di ogni poema presentano ornamentazione ricchissima, con spesse ed elaborate cornici e un sarlauh miniato in ogni caso diverso. Il manoscritto è adorno di 24 miniature di notevole pregio riconducibili allo 'stile di Širāz'. Un esempio eloquente dell'eccezionale finezza del volume già suggerisce l'incipit del primo poema, l'ermetico Mahzan al-asrār, del quale Piemontese curiosamente tace: ai ff. 1v-2r (Figg. 3-4) i primi 8 vv. sono vergati con inchiostro dorato su fondo blu entro due sfaccettati turanğ speculari: qui la sottile nasta'līq quasi a bella posta si confonde tra i racemi egualmente dorati, le corolle di fior rossi e dorati che in qualche caso paiono sbocciare dagli stessi tratti delle lettere; quanto alla disposizione del testo, come si può vedere dalla trascrizione, non è prestata alcuna attenzione alla scansione metrica degli emistichî: i vv. sono disposti come meglio potevano colmare l'area ovale del turanğ; il primo e l'ultimo rigo di ognuno sono da leggersi di séguito, sì da ottenere lo 'unwān: Kitāb-i Hamsah-i šayh Nizāmì 'alàyh' 'r-rahmah; per il resto, i vv. 1-4 si leggono nel primo medaglione, i vv. 5-8 nel secondo. Oltre che per la innegabile bellezza, il manoscritto appare d'interesse anche sotto il profilo testuale: 


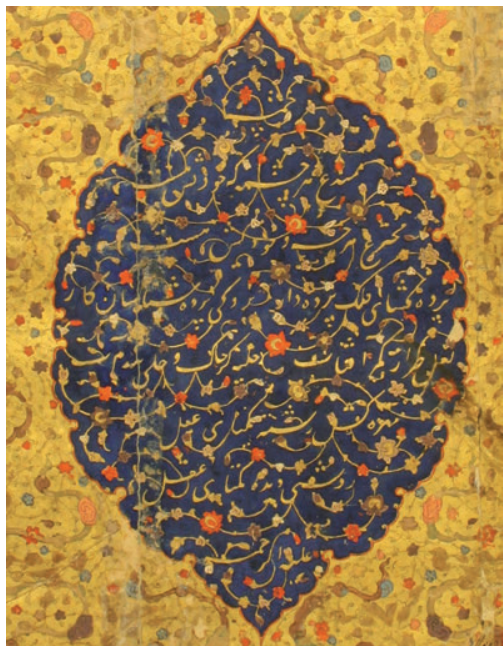

Fig. 3 - Fondo CASTIGLiOni 22, f. 2r.

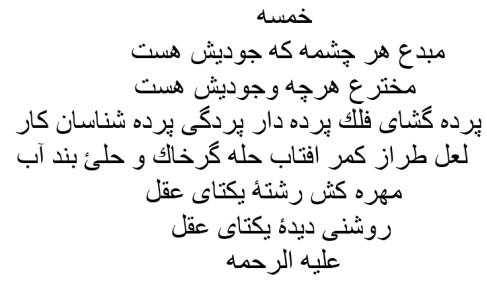

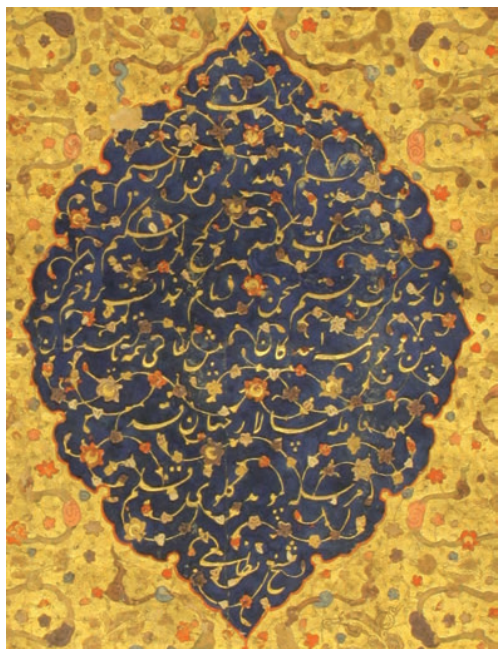

Fig. 4 - Fondo CASTigLioni 22, f. 1v.

$$
\begin{aligned}
& \text { كتاب } \\
& \text { بسم الله الرحمن الرحيم } \\
& \text { هست كليد در كنج حكيم الرحيم }
\end{aligned}
$$

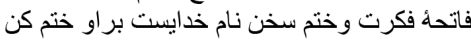

$$
\begin{aligned}
& \text { ييش وجود همه ايندكان بيش بقاى همه بِايندكان }
\end{aligned}
$$

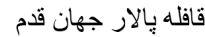

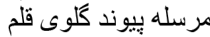

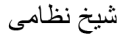

Il primo emistichio di v. 4, qui a 1.6 del medaglione di sinistra reca, ad esempio, invece del nesso قافله سالار رفت قافله سارة, cf. la iunctura adombrante la morte del Profeta, la variante قافله "ِالار: dunque non "condottiero", ma la più concettosa immagine di "trave portante". L'edizione di "Alizzāde non riporta che la variante سابقه dei mss. خ e s.?

Di non piccolo interesse è la composita parte turca del fondo. Dal lascito di Madini proviene AG.XI.43; il corposo volume non è a rigor di termini un manoscritto orientale: esemplato in Francia tra i secc. XVII e XVIII, è un dizionario turco - francese. Una nota sul f. Ir informa di un

7 Nizāāì̄ Ganğawī, Maȟzan al-asrārr, matn-i 'ilmī ū intiqādī ba-sa'ī ū ihtimāmi Ăbdùlğărim Ăli oghlü Ălizadă, Baku, Našriyat-i Farhangistān-i 'Ulūm-i Ğumhūrī-i Sūsiyālīstī-i Azarbūyğān, 1960, 1. 
illustre precedente possessore (forse ne fu anche l'estensore?): 'ex libris Pétis de Lacroix', probabilmente François Pétis de la Croix il giovane (1653-1713), piuttosto che l'omonimo padre (1622-1695). Non si tratta di un semplice lessico di voci turche: per molti lemmi sono forniti accurati esempî di fraseologia. Dal medesimo milieu dell'orientalistica francese tra Sei- e Settecento, quello dei Mercuriales che vedevano riunirsi Antoine Galland, Barthélemy d'Herbelot de Molainville e, appunto, François Pétis de la Croix, proviene con tutta probabilità anche AG.X.39. Si tratta di un quaderno ove sono raccolte trentasei hikāyāt di Hoğa Nașreddīn con traslitterazione, traduzione e commento in francese. Il testo osmanli è vergato in un'elegante nashī dovuta allo stesso estensore.

Ancora dalla biblioteca del Madini proviene la copia del Mă̆ma

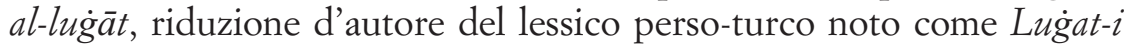
Ni'mat Allāh, dovuto a Ni'mat Allāh b. Ahmmad b. Qāḍ̄ Mubārak, smaltatore oriundo di Sofia; giunto ad Istanbul, fu cooptato nella confraternita naqšbandita, nel cui ambiente acquisì una conoscenza profonda delle lettere persiane. Morì nel 969 èg. $=1561 / 2$ d.C. e fu sepolto nei giardini del convento presso la porta di Edirne. Probabilmente per impulso del dotto consigliere di Selīm e fecondo scrittore nelle lingue turca, persiana ed araba Kemāl Pāšā-zāde (873?-940èg. = 1468?-1534), egli attese alla redazione del proprio dizionario persiano-turco attingendo alla lessicografia più antica senza trascurare la coeva lingua popolare. ${ }^{8}$ Tale attenzione per i demotismi fa del Lugat-i Ni"mat Allăh una preziosa fonte dialettologica ed etnografica: così, ad esempio, al f. 98v, la voce persiana sarhwuš, "inebriato", è glossata anche con voce del turco orientale üsrük ya'nī mest. In tale versione sintetica del lessico, rispetto al ms. di S. Pietroburgo studiato da Otto Blau, alcuni lemmi di particolare interesse dialettologico paiono assai raccorciati. Blau trascrisse, tra l'altro, alcune esaustive glosse di voci attribuite dal lessicografo - con corredo di occorrenze poetiche - al vernacolo di Ișfahān, un particolare omesso nella copia milanese della riduzione: per la voce zūnğ, "salsiccia” ad esempio, il nostro ms., f. 92v, 1. 15, ricorda seccamente: sucuq

8 O. Blau, Ueber Ni'met-ullah's persisch-türkisches Wörterbuch, Zeitschrift der deutschen Morgenländischen Gesellschaft XXXI, (1877), 484-494; E. Berthels, Ni'mat Allab bin Ahmad, EI VIII, 45a; Id., Nimetullah, A IX, 285a-b; C.A. Storey, Persian literature, a bio-bibliographical survey, III.1: A. Lexicography; B. Grammar; C. Prosody and poetics, Leiden, E.J. Brill ltd., 1984, 70 s., nr. 102. 
ya'n̄̄ mumbar, laddove il ms. russo soggiunge Isfahān dilinde mūmbar derler; così, della voce šaftarang, "albicocca", che il ms. russo ugualmente dice usuale di quella città, il nostro ms. offre una ben più asciutta esegesi, f. 115r. 1. 2: şekil-i şeftālū ve-qayısı.

Di grande rarità è AG.X.35, sobrio manoscritto del sec. XVIII in scrittura nashī ad inchiostro nero, completamente vocalizzata, dettaglio di non trascurabile valore per lo studio dell'ortografia e della pronunzia dell'osmanli più antico. Si tratta di una copia del Fetihname-i Hayr ed-dīn Paşa di Seyyid Murādī, secondo Aldo Gallotta, 'scritto indipendentemente dalle $\dot{G} a z a v a \bar{a} t$ in versi e poi aggiunto ad esse in modo da continuarne la narrazione'. Vi si cantano le gesta del Barbarossa in Puglia nel 1537, che culminarono nella battaglia di Prevesa (1538); lo tramandano solamente altri due mss.: Cairo, Dār alkutub, turco 3601 (datato al 1538 e probabilmente autografo) e Istanbul, Topkap1 670 (Revan 1292). ${ }^{9}$

Giunti, al più tardi, con la soppressione napoleonica piuttosto che di pertinenza della preesistente biblioteca gesuitica appaiono due manoscritti turchi recanti l'ex libris della 'Libreria di San Bernardino di Rimini’: AG.XI.21 e AG.XI.22. Una nota sul foglio di guardia di quest'ultimo, un trattato di morale religiosa mutilo del principio, ci informa forse della visita in Braidense di un celebre diplomatico e orientalista piemontese, antesignano della turcologia italiana: Questo libro in lingua turca non è che I un commento sopra un'opera originale I araba il cui argomento vien accennato in fine I del manoscritto [...] Nota del Cavalier Romualdo Tecco Consigliere di legazione I di S.M. Sarda, addi

9 F. Babinger, Die Geschichtsschreiber der Osmanen und ibre Werke, Leipzig, Otto Harrassowitz, 1927, 79; L. Forrer, Handschriften osmanischer Historiker in Istanbul, Der Islam 26, (1942), 173-220: 191, nr. 30; Agâh Sirri Levend, Ġazavāt-nāmeler ve Mihaloğlu Ali Bey'in gazavāt-nāmesi, Ankara, Türk Tarih Kurumu basimevi, 1956 (Türk Tarih Kurumu yayınlarıdan, XI. Seri, no. 8), 70-73; Fehm Edhem Karatay, Topkapı Sarayı Müzesi Kütüphanesi Türkçe yazmalar kataloğu, Istanbul, Topkapı Sarayı Müzesi, 1961 (Topkapı Sarayı Müzesi Yayıları, no. 11), 219, nr. 670; A. Gallotta, I «Ǵazavāt-i Hayreddīn Paşa» di Seyyid Murād, Napoli, Centro di Studi Magrebini, 1981 (Studi Magrebini XIII), 19 ss.; Id., Khayr al-Dīn (Khıdır) Pasha, Barbarossa, EI IV, 1155a-1158b; Fìris al-mahtūțāt at-turkiyyat al-utimāniyyat allatī iqtanatuhā Dār alkutub al-qawmīyah mundu 'àm 1870 hattà nihāyat 1980 m., I [al-Qāhira], al-Hai'at almișriya al-'Āmmat li-'l-kitāb, 1987, 175b-176a, nr. 3601; M. Yildiz, Ġazavāt-i Hayr eddīn Paşa (MS 2639 Universitätsbibliothek Istanbul), kommentierte Edition mit deutscher Zusammenfassung, Aachen, Verlag Shaker, 1993, 21-36: 27 ss. 
27 di genn. 1846. ${ }^{10}$ L'altro manoscritto, AG.XI.21, in rozza scrittura nash̆ $\overline{~ a d}$ inchiostro nero, con frequenti mende ortografiche, è un Corano introdotto dalle consuete orazioni arabe e seguito da qualche pagina di preghiere turche. Al f. $1 \mathrm{r}$ (Fig. 5), tinto di color croco, si legge un'interessante nota:

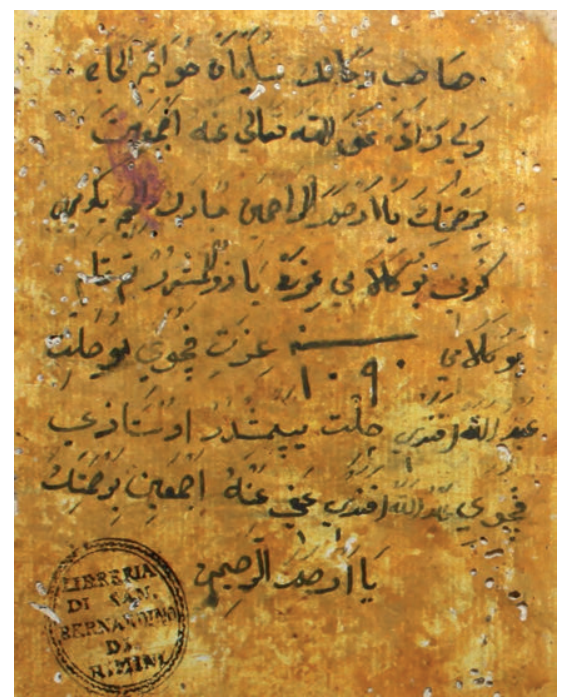

Fig. 5 - AG.XI.21, f. 1r.

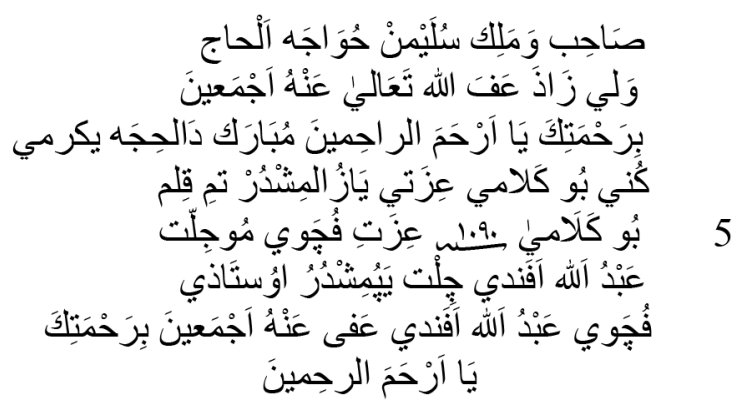

10 Intorno alla figura del Tecco, vd. almeno G. d'Erme, Romualdo Tecco (18021867), diplomatico sardo "orientalista", in Annali della Facoltà di Lingue e Letterature 
Signore e padrone (è) ser Süleymān, che ha compiuto il pellegrinaggio I figlio di Velī, perdonalo, Iddio l'altissimo, di tutto, I per la tua misericordia, o più misericorde tra i misericordi. Nel (mese) benedetto di Dū 'lHiğğah, ventesimo I giorno, questa parola gloriosa è stata scritta: s'è compiuta la scrittura I di questa parola nell'anno 1090. La gloria di Foça, il legatore I messer 'Abdallāh (lo) ha rilegato, il maestro I di Foça messer 'Abdallāh perdonalo, Iddio l'altissimo, di tutto, per la tua misericordia, I o più misericorde tra $i$ misericordi.

Essa informa dei nomi del copista-possessore (11. 1-2: Sạhib vemālik Süleymān hoca el-hâacc| Velìzāde) e perfino di chi attese alla legatura del volume (11. 5-6: mūcellit - cilt yapmışdur) il 22 gennaio 1680 (1l. 3 e 5: mübārek (u) al-hicce yiğirmi I güni - sene 1090), un uomo di nome 'Abdallāh oriundo di Foça, l'antica Focea (11. 5 e 7: foçevi). Del periodo in cui il Corano fu copiato, tra il 15 aprile e il 14 maggio 1676, informa invece il colofone, al f. $338 \mathrm{v}$ :

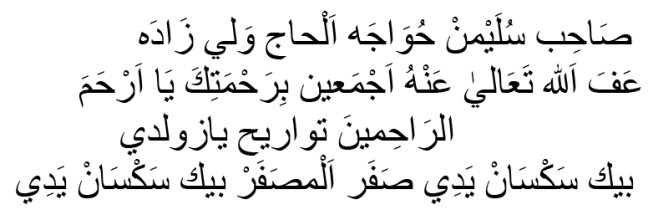

Signore ser Süleymān, che ha compito il pellegrinaggio, figlio di Velī $\mid$ perdonalo, Iddio l'altissimo, di tutto, per la tua misericordia, o più misericorde | tra i misericordi. La data (1. tevārīh) è stata scritta | il mille ottantasette, Safar il trionfante (l. muzaffer) mille ottantasette.

A giudicare dalla fattura del volume, sembra chiaro come Süleymān Hoca non fosse un copista di professione, ma un semplice devoto, forse appartenente a qualche confraternita religiosa, che ricopiò l'intero Corano come esercizio di pietà. Tale profonda dedizione al libro sacro anche traspare in una frase ricalcata in rosso in calce alla pagina:

straniere di Ca' Foscari IX, (1970), Serie orientale, 1, 107-122. In merito ai suoi precipui interessi di studio, riflessi dai volumi che adunò, cf. A. Gallotta, I manoscritti turchi della Biblioteca Reale di Torino, in Studi in onore di Francesco Gabrieli nel suo ottantesimo 


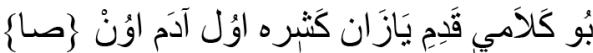

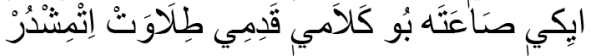

Chi copiò questo Corano, una volta sbiadito (keșere), questo (stesso) uomo in dodici ore ravvivò (tilāvet itmişdür, lett. "abbelli”, scil. la scrittura di) questo Corano. ${ }^{11}$

compleanno, a cura di R. Traini, Roma, Università di Roma "La Sapienza" - Dipartimento di Studi Orientali, 1984, 349-372. sta frase.

11 Debbo alla dotta cortesia D.V. Proverbio la corretta interpretazione di que- 
